\title{
Meta-Heuristics Algorithms: A Survey
}

\author{
Ibrahim El-Henawy \\ Department of computer science, faculty of \\ computers and informatics \\ Zagazig University, \\ Egypt
}

\author{
Nagham Ahmed Abdelmegeed \\ Department of computer science, faculty of \\ computers and informatics \\ Zagazig University, \\ Egypt
}

\begin{abstract}
This paper is meant to present a meta-heuristic algorithms and their application to combinatorial optimization problems. This report contains an assessment of the rapid development of meta-heuristic thoughts, their convergence towards a unified fabric and the richness of potential application in optimization problems. The paper presents a brief survey of different metaheuristic algorithms aiming to solve optimization problems. The meta-heuristic is divided into four broad categories Evolutionary, Physics-based, Swarm-based and Human-based algorithms.
\end{abstract}

\section{Keywords}

Meta-heuristics, algorithms, optimization, evolutionary, Physics-based, swarm-based and human-based algorithms.

\section{INTRODUCTION}

Optimization is the science of picking out the best option among a weakened circle of options [1] or it can be viewed as unitary of the major measurable tools in network of decision making in which determinations have to be hired to optimize single or more grades in some approved set of conditions.

Meta-heuristic algorithms are required for many causes:

i. Support Optimization problem by causing a structure that the cognitive operation of determining the exact solution cannot be fixed.

ii. Meta-heuristic algorithms are a simple tool from the point of decision making

iii. Can be applied as a component of the process of determining the exact solution, and learning purposes.

iv. Leaving us to determine which choices must be proven, how problem's data must be collected) are ignored in the definitions made with mathematical formulas.

Although Meta-heuristic optimization algorithm is the best answer in real life problem the solution of meta-heuristic algorithm is the best solution but not as the optimal solution. Meta-heuristic optimization algorithm is preferable in engineering application according to:

1. Easy to integrate with your existing implementation

2. Not required gradient information

3. Can be applied in a broad range of problem covering different subjects

On that point are four primary categories of Meta-heuristic optimization algorithms: Swarm-based, Human-based, Physics-based, and Phylogeny- based.

The paper divided into three sections, section 1 will represent the Meta-heuristic Basic Categories and their algorithms, section 2 Comparative Study of algorithms and their diligence and final results and conclusion.

\section{META- HEURISTIC BASIC CATEGORIES}

Meta heuristics algorithms includes basic categories shown at figure (1).The First category includes Genetic Algorithms (GA) and popular algorithms such as Evolution Strategy (ES), Probability-Based Incremental Learning (PBIL), Genetic Programming (GP), and Biogeography-Based Optimizer (BBO) [3].

The Second Category are the Physics-based methods imitate the physical principles of the universe include Big-Bang BigCrunch (BBBC), Gravitational Search Algorithm (GSA), Charged System Search (CSS), Central Force Optimization (CFO), Artificial Chemical Reaction Optimization Algorithm (ACROA), Black Hole (BH) algorithm, Ray Optimization (RO) algorithm, Small-World Optimization Algorithm (SWOA) [4]

The third group of nature-inspired methods include swarmbased techniques that simulate the social behavior of groups of animals. The Category of algorithms Particle Swarm Optimization, Teaching, Learning Based Optimization (TLBO), Harmony Search (HS), Tabu (Taboo) Search (TS), Group Search Optimizer (GSO), Imperialist Competitive Algorithm (ICA), League Championship Algorithm (LCA), Firework algorithm, Colliding Bodies Optimization (CBO), Interior Search Algorithm (ISA) [1] [5].

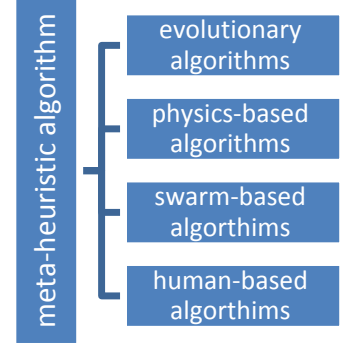

Figure 1: Metaheuristic Basic Categories 


\subsection{Evolutionary algorithms}

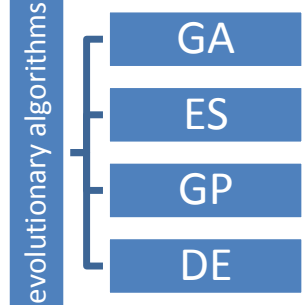

Evolutionary algorithms consists of an important algorithms are listed below at figure (2)

Figure 2: evolutionary algorithms categories

\subsubsection{Genetic Algorithm}

Genetic algorithms have been used to a wide range of practical problems [6], regularly with valuable results. Small examples can effectively explore a large space because they contain schemata; useful substructures that can be potentially combined to make fitter individuals Genetic algorithms have a physical body of different properties:

- Working with parameters encoding.

- Use a fitness function that does not affect the calculation of differential coefficients.

- Search probabilistically.

- They search by substance of a population of people.

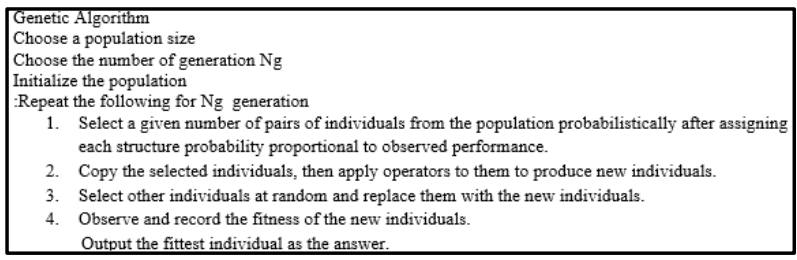

Figure 3: The pseudo code of GA

\subsubsection{Genetic Programming (GP)}

GP is package of computer programs that exemplified as tree structures in memory [7].The main GP five Preparatory Steps are: Includes a bunch of terminals for each branch of the to be evolved program, a set of crude functions ,The fitness measure will have two components, Functions, parameters for the testing process, and Termination criterion and picking result methodology.

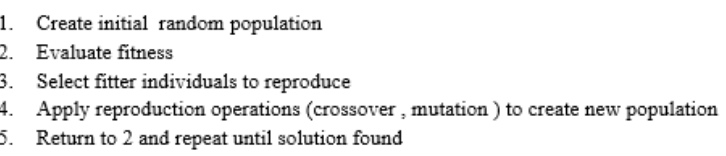

Figure 4: The pseudo code of GP

\subsubsection{Differential Evolution (DE)}

$\mathrm{DE}$ is a random exploration technique and one of global optimization algorithms [8]. It is related the package of Evolutionary Programming, and Evolution Strategies, but has a matches with Particle Swarm Optimization. DE is used for multidimensional real-valued functions don't use the gradient of the problem being improved, which means DE doesn't need the optimization problem to be differentiable as is mandated by classical optimization methods such as gradient descent and quasi-Newton methods.

\subsubsection{Evolution Strategy}

Evaluation Strategy was prepared in the first stage as a method to find optimal parameter settings in laboratory experiments not as an algorithm to be used with computers
[9]. It just focuses on interpreting the underlying mechanisms of biological evolution for technical optimization problems. Evolution Strategy is not founded on detailed simulation of the methods found with natural evolution. Which can already be resolved by seeing at the opposing terms: evolution and strategy? In biological evolution on that point is no strategy, oriented towards a specific goal to be achieved or a special result to be launch.

\subsection{Physics-based algorithms}

Physics-based algorithms consists of an important algorithms are listed below at figure (5).

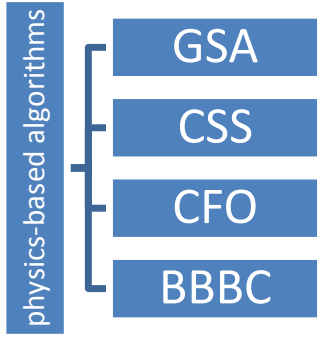

\section{Figure 5: physics-based algorithms categories}

Scientists use several physical [10], chemical, biological laws which are assisting them to improve new optimization methods help in solving of complex, multimodal, high dimensional and nonlinear problems the meta-heuristic optimization methods are employed. Physics based algorithms anticipated by different scientists for deeply exploring the associated literature.

\subsubsection{Gravitational Search Algorithm}

Granting to the gravity law, every atom in the universe attracts each other with a force, this strength is relative to their masses, and inversely proportional to the second power of the length between them from this perspective GSA is inspired based on the law of gravity and laws of motion [11]. In this algorithm, every agent is denoted as objects. The objects' performances are evaluated by their masses. The anticipated results of extending this algorithm the position of the object with the heaviest mass will show the global solution to solve complex optimization system problems. The anticipated results of extending this algorithm the position of the object with the heaviest mass will show the global solution to solve complex optimization system problems.

Step 1: Identify the search space
Step 2: Initialize
Step 3: Evaluate Fitness
Step $4:$ Update best $(t)$, worst $(t)$ and $M(t)$ and $G(t)$
Step $5:$ Calculate total force in different directions
Step $6:$ Calculate acceleration and velocity
Step 7: Update agent's position
Step $8:$ Repeat step 3 to 6 unit the meeting stop criteria

Figure 6: The pseudo code of GSA

Although GSA and PSO gave the same results and same level of recompense for the SVC placement, but GSA quickly found the high quality optimally solution in determining the location and size of SVC. GSA gives good effects as well.

\subsubsection{Central Force Optimization Algorithm}

Central Force Optimization (CFO) Algorithm is a metaheuristic algorithm. Unlike the many stochastic algorithms 
[13]. The CFO is a deterministic technique, which seeks a multi-dimensional decision space. In this algorithm, investigations coast along with the decision planetary under the impression of gravity and they alter their places according to the equations of movement. In process of time, it is anticipated that all investigations will be trapped in close orbits of big messes with largest gravitational field [14]. That means they slowly travel towards the probe that has reached the highest mass or fitness.

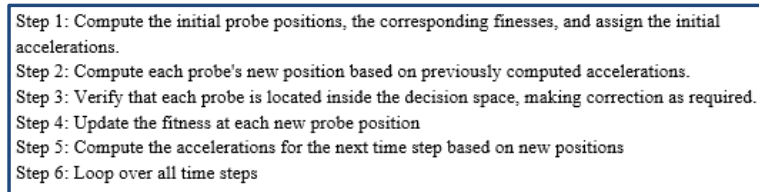

Figure 7: The pseudo code of CFO

\subsubsection{Big Bang-Big Crunch Algorithm ((BB-BC))}

BB-BC algorithm based on the Big Bang and Big Crunch model that is about the phylogeny of the world [15]. The algorithm includes two phases: big-kick and big-crunch]. In the first stage, the algorithm generates an initial random population that is used in big crunch phase. In the big crunch phase, the population decreases to singularity.

Step 1: Form an initial generation of $\mathrm{N}$ candidates in a random manner. Respect the limits
of the search space.
Step 2: Calculate the fitness function values of all the candidate solutions.
Step 3: Find the center of mass.
Step 4: Calculate new candidates around the center of mass by adding or subtracting
normal random number whose values decreases as the iteration elapse.

Figure 8: The pseudo code of BBBC

\subsubsection{Applications of BBBC Algorithm}

The most Applied for BB-BC is born Only Target Motion Analysis (BO-TMA) [16]. The result showed that in BOTMA problem, BB-BC algorithm converged faster than the other common evolutionary computing methods. Alatas integrated chaos and uniform population method to BB-BC algorithm and reported promising effects. The reaching time of the global minima was decreased by this change. BB-BC algorithm also applied to timetabling problem. Experiments proved that the algorithm could find sound quality results [17].

\subsubsection{Charged System Search Algorithm}

In this algorithm, each agent is represented as a Charged Particle (C) [18]. Each molecule is thought as an alternative of the solution. In improver to that, the law of motion is employed to direct other charged atoms with their fitness values and separation distances move the movement of CPs. $\mathrm{CP}$. The power that played on each $\mathrm{CP}$ determines its new location, velocity and acceleration [19]. Figure (9) presents the steps of the algorithm.

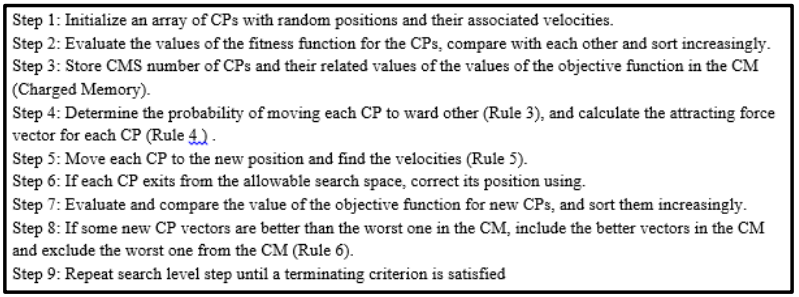

Figure 9: The pseudo code of CSSA

\subsubsection{Applications of CSSA Algorithm}

The First Application of CSSA is an improved discrete version of the CSSA optimize truss structures with discrete variables [20]. The algorithm used several standard examples to compare with the other Meta heuristics and the results indicated that CSSA had better performance than the others. In improver to that CSSA had better convergence than the others proposed methods. After comparing CSSA with some others popular meta-heuristic algorithms. CSSA had better solutions in a less iteration numbers. [21]

\subsection{Swarm-based algorithms}

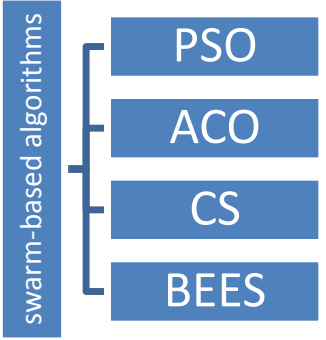

Figure 10: swarm-based algorithms categories

The third Categories of meta-heuristic algorithms are swarmbased algorithms include basic types of algorithms listed at figure (10). This algorithm is based on the nature-inspired behavior and is effectively

Applied to optimization problems in a variety of fields [22]. Swarm algorithm is type of optimization algorithm that used: In machine learning filed, optimization techniques can be applied to determine the parameters for sorting algorithms such as:

- Artificial Neural Networks

- Support Vector Machines

\subsubsection{Particle swarm optimization (PSO)}

PSO shares many similarities with evolutionary computation techniques such as Genetic Algorithms (GA) [23]. In PSO, the potential results, titled as particles, hover through the problem space by following the present optimal particles. The advantages of PSO are that PSO is easy to implement and there are few parameters to set. We can practice it in multiapplication like face recognition, selected features, in mechanical design, in heating system planning problem, in railway domain and in another field.

The PSO algorithm consists of barely three steps:

1) The fitness function for each atom.

2) Update individual and global bests 
3) Position and velocity update.

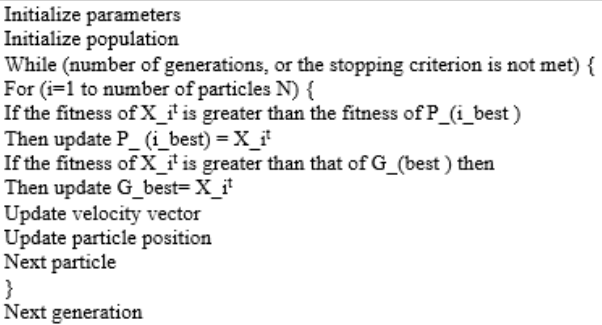

Figure 11: The pseudo code of PSO

We can practice it in multi-application like face recognition, selected features, in mechanical design, in heating system planning problem, in railway domain and in another field.

\subsubsection{Ant Colony Optimization (ACO)}

ACO algorithms are developed and successfully applied to several NP-hard [24]. The inspirational basis of ant colony is the foraging behavior of real ant colonies, This actions used in artificial ant colonies for the search of estimated solutions to discrete and continuous optimization problems and to important problems in telecommunications [examples : routing and load balancing] and also works as a multi-agent approach to difficult combinatorial optimization problems like the TSP and the quadratic assignment problem (QAP).The TSP explained in figure(12) is extensively has attracted since long time a considerable amount of research effort it also plays an important role in Ant Colony Optimization since the first ACO algorithm[15], called Ant System.

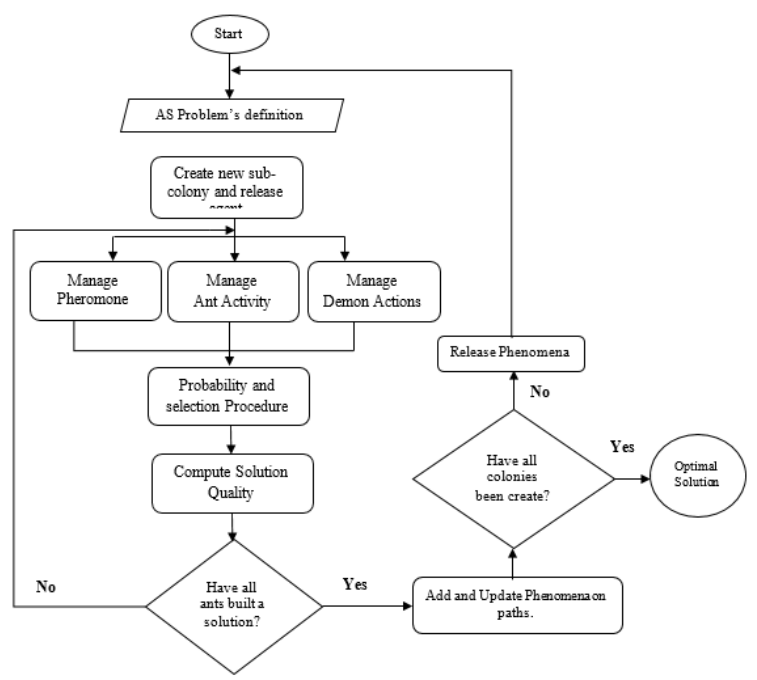

Figure 12: Flowchart of ACO algorithm

\subsubsection{Among the problems that ACO can solve for} example;

* Travel salesman problem.

* Genetic hybrids for the quadratic assignment problem.

* A scheduling problem.

* Timetable problem.
* Graph coloring problems
* Shortest subsequence problem.
* Data mining.
* Multi objective problems.

\subsubsection{Cuckoo search}

Cuckoo search is Hybrid Algorithms that can get the best results and efficiently for the many continuous optimization problems [27]. In some shells, the solutions cannot be institute for some optimization problems. Cuckoo search represents a novel solution for the Meta heuristic optimization problem [27].figure (14) represent the basic steps of the algorithm. The obtained solution is a novel resolution based on the adjustment of some features of the living one. Cuckoo search has three basic principles [26]:

1. Each cuckoo lays one egg [selected element] at a time, and dumps its egg in a randomly chosen nest [target branch].

2. The nests with high quality [high score] of eggs will carry over to the next generation.

3. The number of available host nests is fixed and the host bird finds the egg laid by the cuckoo having fixed probability.

Cuckoo search used in many application like:

- Real-time continuous speech recognition system

- Globle optimization

- Job scheduling

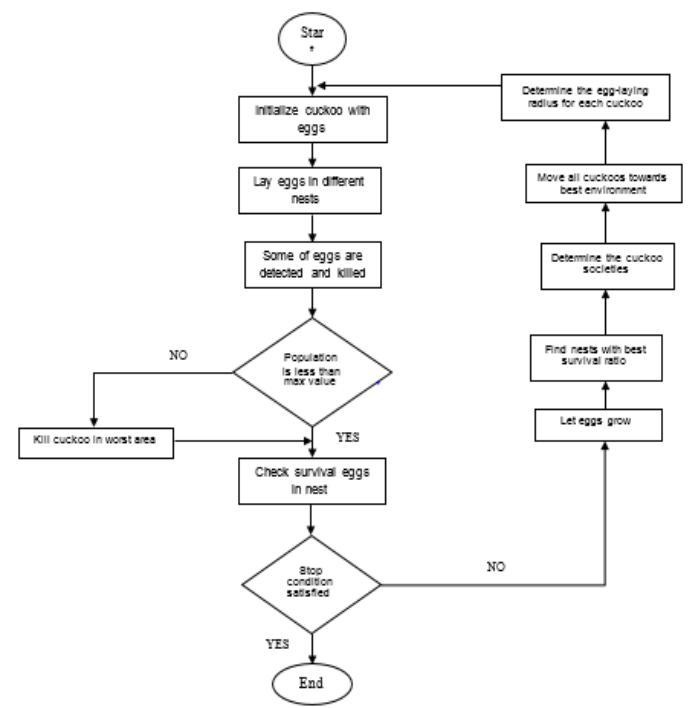

Figure 14: Flowchart of CS algorithm

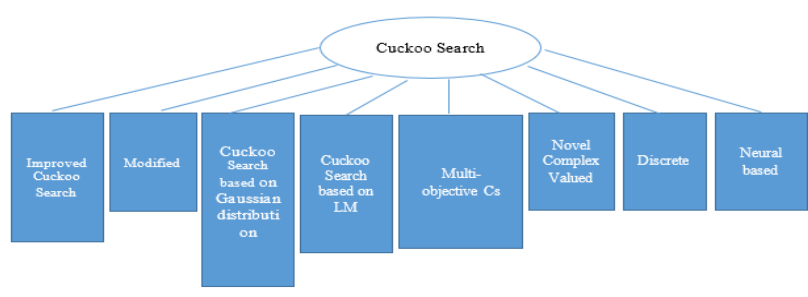

Figure 13: The type of CS algorithm 


\subsubsection{The Bees Algorithm}

BBBS is stimulated by the natural behavior of honeybees throughout the pollen collection process [28]. The process moves up by sending pathfinder to search round for talented sites. Scout bees move randomly during the searching. When they come back to the hive, they show by dancing three pieces of information about found site: the direction in which it is located, its distance from the hive and its quality [29]. This information aids the colony to assess the quantity of energy required to get it and after it, they can send its bees to the most talented place straight. Each individual's acquaintance of the outside environment comes only from this race. After dancing more admirer bees are shipped to more fields that are promising. This allows the colony to return pollen quickly and efficiently.

1) Randomly generates the initial population.

2) Evaluate the fitness for all nominees.3) Repeating of the following steps until a satisfactory solution is reached:

- Select sites for circumambience search.

- Send out bees to selected situations (more bees for better sites) and assess its fitness.

- Select bees with the highest fitness from each patch.

- Allocate residual bees to search randomly and estimate their fitness.

At the conclusion of each repetition has sworn two parts of the population - individuals from selected sides and scout bees designate for searching. Finally the algorithm reaching the satisfactory solution or after giving the number of repeats.

\subsection{Human-based algorithms}

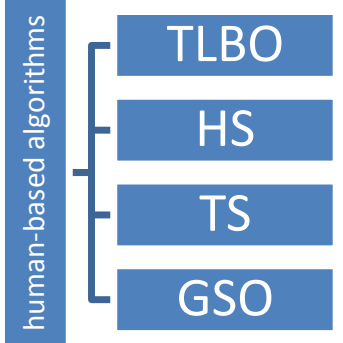

Figure 15. human-based algorithms categories

There are also other meta- heuristic methods inspired by human behaviors shown at figure (15) in the literature. Some of the most popular algorithms are Teaching, Learning Based Optimization (TLBO), Harmony Search (HS), Tabu (Taboo) Search (TS), and Group Search Optimizer (GSO) shown in figure (15) now we explain these most popular algorithm [30].

\subsubsection{The TLBO algorithm}

TLBO algorithms are a teaching-learning process motivated algorithm proposed based on the effect of the influence of a teacher on the output of learners in a class [31]. The algorithm defines two plain approaches of the scholarship:

\section{(i) Teacher Phase}

(ii) Interaction with the other learners (known as lunar phase).

Teaching-learning-based optimization (TLBO) is a population-based algorithm that simulates the teachinglearning process of the classroom. This algorithm needs merely the mutual control parameters such as the population size and the number of peers and does not require any algorithm-specific control parameters [33]. The optimization problem and a learner's outcome are similar to the 'fitness' rate of the optimization problem [32]. The pattern variables are truly the restraints involved in the objective function of the given optimization problem and the best result is the best value of the target function.

\subsubsection{Harmony search (HS)}

Harmony search (HS) is a meta-heuristic algorithm, soft computing algorithm or evolutionary algorithm motivated by the simulating musician's process [34].

It can find best solution as:

- $\quad$ Minimum Cost (Design)

- Minimum Error (Parameter Calibration)

- Maximum Profit (Managerial)

- Maximum Utility (Economics)

This algorithm has more merits like

1. It does not require differential gradients, considering discontinuous functions and continuous functions as the same.

2. Manipulate discrete variables and continuous variables.

3. Does not require initial values for variables.

4. Free from divergence.

Now we show the step of HS that explain how it work

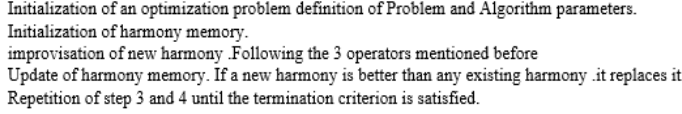

Figure 16: The pseudo code of $\mathrm{HS}$

1. And this is the diligence of this algorithm Optimization Benchmarks, Industry.

2. Power Systems.

3. Digital Signal and Image Processing.

4. Has also been widely used in a great diversity of subjects.

5. Including transportation.

6. Manufacturing.

7. Robotics

8. Control.

9. Medical science. 


\subsubsection{The Group Search}

GS Optimizer the GSO algorithm is based on the ProducerScrounger model inspired by animal searching behavior and is [35]. There are three kinds of member of the group: (1) one producer, search for food; (2) scrounger, perform area copying behavior in order to keep searching for opportunities to join the resources found by the producer; (3) ranger, employs searching strategies of random walks for randomly distributed resources perform the random walk motion. At each iteration, the member located the most promising resource is produced, a number of members except producer in the group are selected as scroungers, and the remaining members are rangers. In GSO, if a member is outside the search space, it will turn back to its previous position in the search space it is quite evident that it is a good optimization algorithm [36]. However, there is quiet an unfit in GSO algorithm concerning the solution search equation, which is used to generate new candidate solutions of GSO algorithm based on the information of previous solutions. The exploration and exploitation are quite necessary for a population-based algorithm. However, they are varying to each other. Hence, in order to achieve satisfactory performance an equilibrium should be found between the two. In this work, we amend the solution search equation by daubing the global best (Gbest) explanation to guide the search of new applicant solutions in order to advance the exploration and exploitation.

\subsubsection{Tabu Search algorithm}

It is a native search-based meta-heuristic algorithm [37]. Tabu Search is fully based on the designation of the neighborhood and activities converting a solution to its neighboring solutions. This algorithm takes up with a solitary solution, and quests for enhanced results, using actions and moving between neighbor solutions. However, approval, applicability and obtainability of actions, are managed using a set of instructions [38]. One of most significant procedures used in Tabu Search, is that: when action is performed, it will be not offered, until a secret sum of other actions achieved.

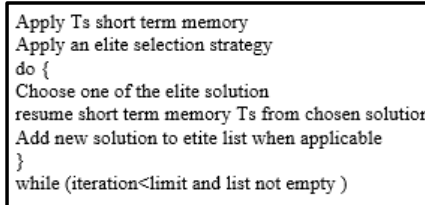

Figure 17: The pseudo code of TS

Extensive achievements in practical applications of optimization have spurred a rapid increment of the method as a way of placing extremely high quality solutions professionally [39]. TS approaches create hybrid actions with other heuristic and algorithmic techniques, to provide more serious answers to problems in "scheduling, sequencing, resource allocation, investment planning, telecommunications and many other applications".

\subsubsection{Applications and architectures: \\ - Scheduling \\ - Telecoms}

- Plan

- $\quad$ Production, Inventory and Investment

- Routing

- Logic and Artificial Intelligence

- Graph Optimization

- Location and Allocation

- General Combinational Optimization

- Technology

Table 1: Meta-heuristic algorithms and their application

\begin{tabular}{|c|c|}
\hline Algorithm & Application \\
\hline GA & $\begin{array}{llr}\text { - } & \begin{array}{l}\text { Bioinformatics: } \\
\text { sequence alignment }\end{array} & \text { multiple } \\
\text { - } & \begin{array}{l}\text { Bioinformatics: } \\
\text { structure prediction }\end{array} \\
\text { - } & \begin{array}{l}\text { Image processing: } \\
\text { pixel matching }\end{array}\end{array}$ \\
\hline ES & 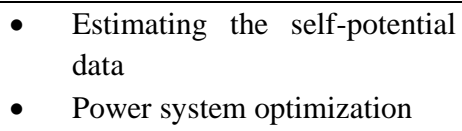 \\
\hline $\mathrm{DE}$ & $\begin{array}{ll}\text { - } & \text { Trading strategy } \\
\text { - } & \text { Technical analysis } \\
\text { - } & \text { Financial indicator } \\
\text { - } & \text { Template evolutionary } \\
& \text { computation }\end{array}$ \\
\hline GSA & 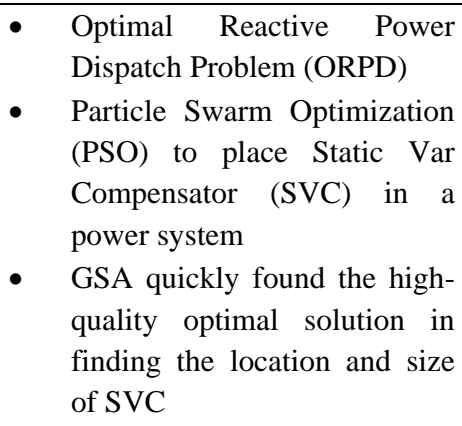 \\
\hline CSS & 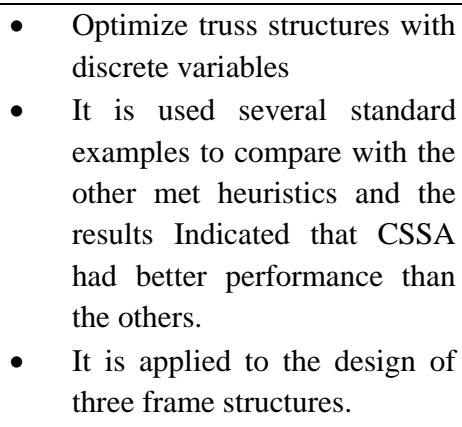 \\
\hline $\mathrm{CFO}$ & $\begin{array}{ll}\text { - } & \text { Face recognition } \\
\text { - } & \text { Selected feature } \\
\text { - } & \text { Sequential ordering } \\
\text { - } & \text { Constraint satisfaction } \\
& \text { problem }\end{array}$ \\
\hline
\end{tabular}




\begin{tabular}{|c|c|}
\hline BBBC & $\begin{array}{l}\text { - It is applied bearing Only } \\
\text { Target Motion Analysis. } \\
\text { BB-BC algorithm converged } \\
\text { faster than the other common } \\
\text { evolutionary computing } \\
\text { methods. } \\
\text { It used to solve the } \\
\text { optimization problem. }\end{array}$ \\
\hline PSO & $\begin{array}{ll}\text { - } & \text { Face recognition } \\
\text { - } & \text { Selected features } \\
\text { - } & \text { Mechanical design } \\
\text { - } & \text { Heating system planning } \\
& \text { problem } \\
\text { - } & \text { Railway domain }\end{array}$ \\
\hline $\mathrm{ACO}$ & $\begin{array}{ll}\text { - } & \text { Travel salesman problem } \\
\text { - } & \text { Quadratic assignment problem } \\
\text { - } & \text { A scheduling problem } \\
\text { - } & \text { Vehicle routing problem } \\
\text { - } & \text { Timetable problem } \\
\text { - } & \text { Shortest super sequence } \\
\text { - } & \text { problem } \\
\text { - } & \text { Consuential ordering } \\
& \text { problem }\end{array}$ \\
\hline BEES & $\begin{array}{ll}\text { - } & \text { Function Optimisation } \\
\text { - } & \text { Electronic Design } \\
\text { - } & \text { BA for TSP } \\
\text { - } & \text { Training NN classifiers like } \\
& \text { MLP, LVQ, RBF and SNNs } \\
\text { - } & \text { Mechanical designs } \\
\text { - } & \text { Digital Filter Optimisation } \\
\text { - } & \text { Fuzzy Control Design }\end{array}$ \\
\hline TLBO & $\begin{array}{ll}\text { - } & \text { Electrical engineering } \\
\text { - } & \text { Mechanical design } \\
\text { - } & \text { Thermal engineering } \\
\text { - } & \text { Manufacturing engineering } \\
\text { - } & \text { Civil engineering } \\
\text { - } & \text { Structural engineering } \\
\text { - } & \text { Computer engineering } \\
\text { - } & \text { Electronics engineering }\end{array}$ \\
\hline HS & $\begin{array}{ll}- & \text { Optimization } \\
& \text { Benchmarks, Industry, } \\
\text { - } & \text { Power Systems } \\
\text { - } & \text { Signal and Image Processing } \\
\text { - } & \text { Has also been widely } \\
& \text { employed in a large variety of } \\
& \text { fields, } \\
\text { - } & \text { Including transportation, } \\
\text { - } & \text { Manufacturing, } \\
\text { - } & \text { Robotics } \\
-\quad & \text { Control } \\
- & \text { Medical science } \\
\end{array}$ \\
\hline
\end{tabular}

\begin{tabular}{|c|c|}
\hline $\mathrm{TS}$ & $\begin{array}{ll}\text { - } & \text { Scheduling } \\
\text { - } & \text { Telecommunications } \\
\text { - } & \text { Design } \\
\text { - } & \text { Production, Inventory and } \\
& \text { Investment } \\
\text { - } & \text { Routing } \\
\text { - } & \text { Logic and Artificial } \\
& \text { Intelligence } \\
\text { - } & \text { Graph Optimization } \\
\text { - } & \text { Location and Allocation } \\
\text { - } & \text { General Combinational } \\
& \text { Optimization } \\
\text { - } & \text { Technology }\end{array}$ \\
\hline GSO & $\begin{array}{ll}\text { - } & \text { It is used to investigate the } \\
\text { plane and space truss } \\
\text { structures with continuous } \\
\text { variables } \\
\text { - } & \text { Structural Design } \\
\text { - } & \text { Mechanical design } \\
\text { optimization problems }\end{array}$ \\
\hline
\end{tabular}

\section{REFERENCES}

[1] Greco, Salvatore, J. Figueira, and M. Ehrgott. "Multiple criteria decision analysis." Springer's International series (2005).

[2] Lee, Kang Seok, and Zong Woo Geem. "A new metaheuristic algorithm for continuous engineering optimization: harmony search theory and practice." Computer methods in applied mechanics and engineering 194.36 (2005): 3902-3933.

[3] Mirjalili, Seyedali, and Andrew Lewis. "The whale optimization algorithm." Approaches in Engineering Software 95 (2016): 51-67.

[4] Xie, Liping, et al. "Artificial physics optimisation: a brief survey." International Journal of Bio-Inspired Computation 2.5 (2010): 291-302.

[5] Binitha, S., and S. Siva Sathya. "A survey of bio inspired optimization algorithms." International Journal of Soft Computing and Engineering 2.2 (2012): 137151.

[6] Haupt, Randy L., and Sue Ellen Haupt. Practical genetic algorithms. John Wiley \& Sons, 2004.

[7] Nordin, Peter, Wolfgang Banzhaf, and Frank D. Francone. "Efficient evolution of machine code for CISC architectures using instruction blocks and homologous crossover." Advances in genetic programming 3 (1999): 275-299.

[8] Goldberg, David E., and Jon Richardson. "Genetic algorithms with sharing for multimodal function optimization." Genetic algorithms and their applications: Proceedings of the Second International Conference on Genetic Algorithms. Hillsdale, NJ: Lawrence Erlbaum, 1987.

[9] Bäck, Thomas, David B. Fogel, and Zbigniew Michalewicz, eds. Evolutionary computation 1: Basic algorithms and operators. Vol. 1. CRC press, 2000. 
[10] Beheshti, Zahra, and Siti Mariyam Hj Shamsuddin. "A review of population-based meta-heuristic algorithms." Int. J. Adv. Soft Comput. Appl 5.1 (2013): 1-35.

[11] Rashedi, Esmat, Hossein Nezamabadi-Pour, and Saeid Saryazdi. "GSA: a gravitational search algorithm." Information sciences 179.13 (2009): 2232-2248.

[12] Ghasemi, Mojtaba, et al. "Solving optimal reactive power dispatch problem using a novel teachinglearning-based optimization algorithm." Engineering Applications of Artificial Intelligence 39 (2015): 100108.

[13] Xing, Bo, and Wen-Jing Gao. Innovative computational intelligence: a rough guide to 134 clever algorithms. Switzerland: Springer International Publishing, 2014.

[14] Wiener, Norbert. Cybernetics or Control and Communication in the Animal and the Machine. Vol. 25. MIT press, 1961.

[15] Labbi, Y., and D. Attous. "BIG BANG-BIG CRUNCH OPTIMIZATION ALGORITHM FOR ECONOMIC DISPATCH WITH VALVE-POINT EFFECT."Journal of Theoretical \& Applied Information Technology 16 (2010).

[16] Genc, H. M., and A. K. Hocaoglu. "Bearing-only target tracking based on big bang-big crunch algorithm." Computing in the Global Information Technology, 2008. ICCGI'08. The Third International MultiConference on. IEEE, 2008.

[17] Can, Umit, and Bilal Alatas. "Physics based metaheuristic algorithms for global optimization." American Journal of Information Science and Computer Engineering 1.3 (2015): 94-106.

[18] Kaveh, Ali, and Siamak Talatahari. "Optimal design of skeletal structures via the charged system search algorithm." Structural and Multidisciplinary Optimization 41.6 (2010): 893-911

[19] Kaveh, A., and S. Talatahari. "Hybrid charged system search and particle swarm optimization for engineering design problems." Engineering Computations 28.4 (2011): 423-440

[20] Can, Umit, and Bilal Alatas. "Physics based metaheuristic algorithms for global optimization." American Journal of Information Science and Computer Engineering 1.3 (2015): 94-106.

[21] Kaveh, A., and S. Talatahari. "Hybrid charged system search and particle swarm optimization for engineering design problems." Engineering Computations 28.4 (2011): 423-440.

[22] Kaveh, A., and S. Talatahari. "Hybrid charged system search and particle swarm optimization for engineering design problems." Engineering Computations 28.4 (2011): 423-440.

[23] Juang, Chia-Feng. "A hybrid of genetic algorithm and particle swarm optimization for recurrent network design." IEEE Transactions on Systems, Man, and Cybernetics, Part B (Cybernetics) 34.2 (2004): 9971006.

[24] combinatorial optimization problems.it is a technique for optimization that was introduced in the early
1990's[Dorigo, Marco, Mauro Birattari, and Thomas Stutzle. "Ant colony optimization."IEEE computational intelligence magazine 1.4 (2006): 28-39.

[25] combinatorial optimization problems.it is a technique for optimization that was introduced in the early 1990's[Dorigo, Marco, Mauro Birattari, and Thomas Stutzle. "Ant colony optimization."IEEE computational intelligence magazine 1.4 (2006): 28-39.

[26] Yang, Xin-She. "Cuckoo search and firefly algorithm: overview and analysis."Cuckoo Search and Firefly Algorithm. Springer International Publishing, 2014. 126

[27] Gandomi, Amir Hossein, Xin-She Yang, and Amir Hossein Alavi. "Cuckoo search algorithm: a metaheuristic approach to solve structural optimization problems." Engineering with computers 29.1 (2013): 17-35.

[28] Pham, D. T., et al. "The bees algorithm-A novel tool for complex optimisation."Intelligent Production Machines and Systems-2nd I* PROMS Virtual International Conference (3-14 July 2006). sn, 2011.

[29] Agazadeh, F., and M. R. Meybodi. "Cooperative Bees Algorithm." International Conference on Measurement and Control Engineering 2nd (ICMCE 2011). ASME Press, 2011.

[30] Mirjalili, Seyedali, and Andrew Lewis. "The whale optimization algorithm."Advances in Engineering Software 95 (2016): 51-67.

[31] Rao, R. Venkata, and V. D. Kalyankar. "Parameter optimization of modern machining processes using teaching-learning-based optimization algorithm."Engineering Applications of Artificial Intelligence 26.1 (2013): 524-531.

[32] Dueck, Gunter, and Tobias Scheuer. "Threshold accepting: a general purpose optimization algorithm appearing superior to simulated annealing." Journal of computational physics 90.1 (1990): 161-175.

[33] Rao, R. Venkata, and Vivek Patel. "An improved teaching-learning-based optimization algorithm for solving unconstrained optimization problems."Scientia Iranica 20.3 (2013): 710-720.

[34] Geem, Zong Woo, Joong Hoon Kim, and Gobichettipalayam Vasudevan Loganathan. "A new heuristic optimization algorithm: harmony search."simulation 76.2 (2001): 60-68.

[35] He, Shan, Q. Henry Wu, and J. R. Saunders. "Group search optimizer: an optimization algorithm inspired by animal searching behavior." IEEE transactions on evolutionary computation 13.5 (2009): 973-990.

[36] Gates, Kathleen M., and Peter CM Molenaar. "Group search algorithm recovers effective connectivity maps for individuals in homogeneous and heterogeneous samples." Neuroimage 63.1 (2012): 310-319.

[37] Glover, Fred, James P. Kelly, and Manuel Laguna. "Genetic algorithms and tabu search: hybrids for optimization." Computers \& Operations Research 22.1 (1995): 111-134. 
[38] Voß, Stefan, et al., eds. Meta-heuristics: Advances and trends in local search paradigms for optimization. Springer Science \& Business Media, 2012.

[39] Gallego, Ramon A., Rubén Romero, and Alcir J. Monticelli. "Tabu search algorithm for network synthesis." IEEE Transactions on Power Systems 15.2 (2000): 490-495.

[40] Abdel-Raouf, O., and Abdel-Baset, M. (2014). A new hybrid flower pollination algorithm for solving constrained global optimization problems. International Journal of Applied Operational Research-An Open Access Journal, 4(2), 1-13.

[41] Abdel-Raouf, Osama, Ibrahim El-Henawy, and Mohamed Abdel-Baset. "A novel hybrid flower pollination algorithm with chaotic harmony search for solving sudoku puzzles." International Journal of Modern Education and Computer Science 6.3 (2014): 38.

[42] Abdel-Baset, M., and Hezam, I. M. (2015). An improved flower pollination algorithm for ratios optimization problems. Applied Mathematics \& Information Sciences Letters An International Journal, 3(2), 83-91.

[43] Abdel-Baset, M., and Hezam, I. M. (2015). An Effective Hybrid Flower Pollination and Genetic Algorithm for Constrained Optimization Problems. Advanced Engineering Technology and Application An International Journal, 4, 27-27.

[44] Abdel-Baset, M., and Hezam, I. (2016). Cuckoo Search and Genetic Algorithm Hybrid Schemes for Optimization Problems. Appl. Math, 10(3), 1185-1192.

[45] Abdel-Basset, M., Hessin, A. N., and Abdel-Fatah, L. (2016). A comprehensive study of cuckoo-inspired algorithms. Neural Computing and Applications, 1-17.

[46] Abdel-Baset, M., and Hezam, I. (2016). A Hybrid Flower Pollination Algorithm for Engineering Optimization Problems. International Journal of Computer Applications, 140(12).

[47] Abdel-Baset, M., and Hezam, I. M. (2016). A hybrid flower pollination algorithm for solving ill-conditioned set of equations. International Journal of Bio-Inspired Computation, 8(4), 215-220.

[48] Abdel-Baset, M., and Hezam, I. M. (2016). Solving Linear Least Squares Problems Based on Improved Cuckoo Search Algorithm.

[49] Metwalli, M. A. B., \& hezam, I. (2015). A Modified Flower Pollination Algorithm for Fractional Programming Problems. International Journal of Intelligent Systems and Applications in Engineering, 3(3).

[50] Hezam, I. M., Abd-ElBaset, M., \& Selem, I. (2015). Cuckoo Search Algorithm for Stellar Population Analysis of Galaxies. International Journal of Information Technology and Computer Science, 7, 2933.

[51] Metwalli, M. A. B., \& hezam, I. (2015). A Modified Flower Pollination Algorithm for Fractional Programming Problems. International Journal of Intelligent Systems and Applications in Engineering, $3(3)$.
[52] Baset, M. A., Satar, M. M. A., Abdel-Raouf, O., \& ElHenawy, I. (2016). Non-Dominated Sorting Genetic Algorithm Based on Altruism for Solving MultiObjective Optimization Problems. Journal of Computational and Theoretical Nanoscience, 13(8), 5060-5071.

[53] Abdel-Raouf, O., and Metwally, M. A. B. (2013). A survey of harmony search algorithm. International Journal of Computer Applications, 70(28).

[54] Abdel-Raouf, O., Abdel-Baset, M., and El-Henawy, I. (2014). An improved chaotic bat algorithm for solving integer programming problems. International Journal of Modern Education and Computer Science, 6(8), 18.

[55] Abdel-Raouf, O., El-henawy, I., and Abdel-Baset, M. (2014). chaotic Harmony Search Algorithm with Different Chaotic Maps for Solving Assignment Problems. International Journal of Computer Applications, 86(10).

[56] Abdel-Raouf, O., Abdel-Baset, M., and El-henawy, I. (2014). Chaotic firefly algorithm for solving definite integral. International Journal of Information Technology and Computer Science (IJITCS), 6(6), 19.

[57] Abdel-Raouf, O., Abdel-Baset, M., and El-henawy, I. (2014). Improved Harmony Search with Chaos for Solving Linear Assignment Problems. International Journal of Intelligent Systems and Applications, 6(5), 55.

[58] El-henawy, I., Abdel-Raouf, O., and Abdelbaset, M. (2014). Improved harmony search algorithm with chaos for solving definite integral. International Journal of Operational Research, 21(2), 252-261.

[59] Wu, H., Zhou, Y., Luo, Q., and Basset, M. A. (2016). Training Feedforward Neural Networks Using Symbiotic Organisms Search Algorithm. Computational Intelligence and Neuroscience, 2016.

[60] Abdel-Baset, Mohamed, et al. "Stellar population analysis of galaxies based on improved flower pollination algorithm." International Journal of Mathematical Modelling and Numerical Optimisation 8.3 (2018): 183-196.

[61] Abdel-Basset, Mohamed, et al. "Feature and Intensity Based Medical Image Registration Using Particle Swarm Optimization." Journal of medical systems 41.12 (2017): 197.

[62] Abdel-Basset, Mohamed, et al. "A modified flower pollination algorithm for the multidimensional knapsack problem: human-centric decision making." Soft Computing (2017): 1-19.

[63] Zhou, Yongquan, et al. "Discrete greedy flower pollination algorithm for spherical traveling salesman problem." Neural Computing and Applications (2017): $1-16$.

[64] Zhou, Yongquan, et al. "A simplex method-based social spider optimization algorithm for clustering analysis." Engineering Applications of Artificial Intelligence 64 (2017): 67-82.

[65] Abdel-Basset, Mohamed, et al. "A comparative study of cuckoo search and flower pollination algorithm on solving global optimization problems." Library Hi Tech 35.4 (2017): 595-608.

[66] Abdel-Basset, Mohamed, et al. "Solving 0-1 knapsack problems by binary dragonfly algorithm." International 
International Journal of Computer Applications (0975 - 8887)

Volume 179 - No.22, February 2018

Conference on Intelligent Computing. Springer, Cham, 2017.

[67] Abdel-Basset, Mohamed, et al. "Krill herd algorithm based on cuckoo search for solving engineering optimization problems." Multimedia Tools and Applications (2017): 1-24.

[68] Abdel-Baset, Mohamed, et al. "Elite opposition-flower pollination algorithm for quadratic assignment problem." Journal of Intelligent \& Fuzzy Systems 33.2 (2017): 901-911.

[69] Abdel-Baset, Mohamed, Haizhou Wu, and Yongquan Zhou. "A complex encoding flower pollination algorithm for constrained engineering optimisation problems." International Journal of Mathematical Modelling and Numerical Optimisation 8.2 (2017): $108-126$ 